\title{
Consistency, population solidarity, and egalitarian solutions for TU-games
}

\author{
René van den Brink ${ }^{1}$. Youngsub Chun ${ }^{2}$. \\ Yukihiko Funaki ${ }^{3}$. Boram Park ${ }^{4}$
}

(c) The Author(s) 2016. This article is published with open access at Springerlink.com

\begin{abstract}
A (point-valued) solution for cooperative games with transferable utility, or simply TU-games, assigns a payoff vector to every TU-game. In this paper we discuss two classes of equal surplus sharing solutions. The first class consists of all convex combinations of the equal division solution (which allocates the worth of the 'grand coalition' consisting of all players equally over all players) and the center-of-gravity of the imputation-set value (which first assigns every player its singleton worth and then allocates the remainder of the worth of the grand coalition, $N$, equally over all players). The second class is the dual class consisting of all convex combinations of the equal division solution and the egalitarian non-separable contribution value (which
\end{abstract}

Y. Chun's work was supported by the National Research Foundation of Korea Grant funded by the Korean Government (NRF-2013S1A3A2055391), and the Netherlands Organization for Scientific Research (NWO) Grant 040.11.143.

$凶$ René van den Brink

jrbrink@feweb.vu.nl

Youngsub Chun

ychun@snu.ac.kr

Yukihiko Funaki

funaki@waseda.jp

Boram Park

borampark@ajou.ac.kr

1 Department of Econometrics, Tinbergen Institute, VU University, De Boelelaan 1105, 1081 HV Amsterdam, The Netherlands

2 Department of Economics, Seoul National University, Seoul 151-746, Korea

3 School of Political Science and Economics, Waseda University, 1-6-1 Nishi-Waseda, Shinjuku-Ku, Tokyo 169-8050, Japan

4 Department of Mathematics, Ajou University, Suwon 443-749, Korea 
first assigns every player its contribution to the 'grand coalition' and then allocates the remainder equally over all players). We provide characterizations of the two classes of solutions using either population solidarity or a reduced game consistency in addition to other standard properties.

Keywords TU-game $\cdot$ Equal division solution $\cdot$ CIS-value $\cdot$ ENSC-value $\cdot$ Population solidarity · Consistency

\section{JEL Classification C71}

\section{Introduction}

A situation in which a finite set of players can obtain certain payoffs by cooperation can be described by a cooperative game with transferable utility, shortly TU-game. A (point-valued) solution on a class of TU-games assigns a payoff vector to every game in the class.

Recently, egalitarian or equal surplus sharing solutions gained attention in the literature. Three well-known equal surplus sharing solutions are the equal division solution (axiomatized in van den Brink 2007), the center-of-gravity of the imputation-set value, and the egalitarian non-separable contribution value. The equal division solution allocates the worth of the 'grand coalition' (being the coalition consisting of all players) equally among all players. The center-of-gravity of the imputation-set value, shortly denoted by the CIS-value (see Driessen and Funaki 1991) first gives every agent its own singleton worth and distributes the remainder equally among all players. The egalitarian non-separable contribution value (also known as equal allocation of nonseparable costs), shortly denoted by the ENSC-value, is the dual of the CIS-value. In van den Brink and Funaki (2009) the class of all convex combinations of these three solutions is studied.

Chun and Park (2012) characterize the CIS-value by efficiency, covariance, and population solidarity, the last property requiring that upon the arrival of a new player all the original players should be affected in the same direction, all weakly gain or all weakly lose. We show that all convex combinations of the equal division solution and the CIS-value satisfy population solidarity and extend the characterization of the CIS-value by Chun and Park (2012) to this class of solutions.

A main goal of axiomatizing solutions is to compare different solutions based on their characterizing properties. In particular, in this paper we consider classes of different equal surplus sharing solutions. On the one hand, by characterizing such a class of solutions we can compare this class with other solutions. On the other hand, to compare solutions within this class, we will consider axioms that depend on the specific parameter determining a specific solution in this class.

Besides axiomatizing all convex combinations of the equal division solution and the CIS-value using population solidarity, we reconsider the axiomatizations using consistency provided by van den Brink and Funaki (2009). They axiomatized the class of all convex combinations of the equal division solution, the CIS-value, and the ENSC-value using a parameterized standardness for two-player games and a para- 
meterized consistency. The convex combinations of the equal division solution and the CIS-value have a nonparameterized consistency in common which we use in an axiomatization together with $\alpha$-standardness for two-player games. For a fraction $\alpha \in[0,1], \alpha$-standardness for two-player games states that for two-player games each player first receives a fraction $\alpha$ of its singleton worth, and what remains of the worth of the 'grand coalition' is split equally among the two players. In van den Brink and Funaki (2009) it is shown that any solution that satisfies efficiency, symmetry, and linearity on the class of two-player games satisfies $\alpha$-standardness for two-player games for some $\alpha \in[0,1]$. Since linearity is only used for two-player games, and in many (economic) applications it is a technical axiom, we prefer to have a characterization of $\alpha$-standardness without linearity. On the other hand, in their characterization of the CIS-value, Chun and Park (2012) use covariance. However, the CIS-value is the only covariant solution in the class considered here. Therefore, we consider a weak covariance which requires that the payoffs of all players change by the same amount if to each coalition we add a constant times the number of players in the coalition. This property can also be seen as a weakening of the fairness axiom in van den Brink (2001). In this paper, we also use another weakening of fairness requiring that the payoffs of all players change by the same amount when we only change the worth of the 'grand coalition'. We show that any solution on the class of two-player games that satisfies these two properties together with efficiency, homogeneity, local monotonicity, and nonnegativity, satisfies $\alpha$-standardness for some $\alpha \in[0,1]$.

To select a particular solution from the convex combinations of the equal division solution and the CIS-value, we use $\alpha$-individual rationality requiring that for appropriate games a player always earns at least a fraction $\alpha \in[0,1]$ from its singleton worth. For specific values of $\alpha$ (1, respectively 0$)$, this axiom yields the usual individual rationality or nonnegativity.

Finally, we introduce a dual version of population solidarity that is satisfied by the ENSC-value, the equal division solution, and all their convex combinations.

The paper is organized as follows: Sect. 2 discusses some preliminaries on TUgames and solutions. In Sect. 3, we consider two-player games and characterize $\alpha$ standardness for two-player games. In Sect. 4, we extend these definitions to $n$-player games using consistency. In Sect. 5, we give an axiomatic characterization using population solidarity. In Sect. 6 we consider the dual class consisting of all convex combinations of the equal division solution and the ENSC-value. Finally, Sect. 7 contains some concluding remarks.

\section{Preliminaries}

A cooperative game with transferable utility, shortly TU-game, is a pair $(N, v)$, where $N \subset \mathbb{N}$ is a finite set of players with $|N| \geq 2$, and $v: 2^{N} \rightarrow \mathbb{R}$ is a characteristic function on $N$ such that $v(\emptyset)=0$. For any coalition $S \subseteq N, v(S)$ is called the worth of coalition $S$. This is what the members of coalition $S$ can obtain by agreeing to cooperate. We denote the class of all TU-games by $\mathcal{G}$. For a fixed player set $N$, we denote the class of all TU-games $(N, v)$ by $\mathcal{G}^{N}$. 
A payoff vector of game $(N, v)$ is an $|N|$-dimensional real vector $x \in \mathbb{R}^{N}$, which represents a distribution of the payoffs that can be earned by cooperation over the individual players. A (point-valued) solution on a class of TU-games $\mathcal{C} \subseteq \mathcal{G}$ is a function $\psi$ which assigns a payoff vector $\psi(N, v) \in \mathbb{R}^{N}$ to every TU-game $(N, v) \in$ $\mathcal{C}$. If a solution assigns to every game a payoff vector that exactly distributes the worth of the 'grand coalition' $N$, then the solution is efficient. ${ }^{1}$ In this paper we discuss two classes of solutions for TU-games that all have some egalitarian flavour. A main reason to consider this class of solutions is that we are interested in equal division solutions but, depending on the application, players might have the right on part of their singleton worth.

The equal division solution ED distributes the worth of the 'grand coalition' equally among all players, i.e., for all $(N, v) \in \mathcal{G}$ and $i \in N$,

$$
\operatorname{ED}_{i}(N, v)=\frac{1}{|N|} v(N)
$$

The center-of-gravity of the imputation-set value CIS, shortly called CIS-value, first assigns to every player its individual worth and distributes the remainder of the worth of the 'grand coalition' $N$ equally among all players, i.e., for all $(N, v) \in \mathcal{G}$ and $i \in N$,

$$
\operatorname{CIS}_{i}(N, v)=v(\{i\})+\frac{1}{|N|}\left(v(N)-\sum_{j \in N} v(\{j\})\right) .
$$

In this paper, we are mainly interested in convex combinations of the equal division solution and the CIS-value, i.e., for every $\alpha \in[0,1]$, the corresponding solution is defined by

$$
\varphi^{\alpha}(N, v)=\alpha \operatorname{CIS}(N, v)+(1-\alpha) \operatorname{ED}(N, v) .
$$

We denote the class of all solutions that are obtained in this way by $\Phi:=\left\{\varphi^{\alpha} \mid \alpha \in\right.$ $[0,1]\}$. It is straightforward to verify that for every $(N, v) \in \mathcal{G}$, and every $\alpha \in[0,1]$ and every $i \in N$, it holds that

$$
\varphi_{i}^{\alpha}(N, v)=\alpha v(\{i\})+\frac{1}{|N|}\left(v(N)-\sum_{j \in N} \alpha v(\{j\})\right)
$$

Alternatively, we can define each solution $\varphi^{\alpha}$ as the CIS-value of a modified game. For $\alpha \in[0,1]$ and $(N, v) \in \mathcal{G}$, define the game $\left(N, w^{\alpha}\right)$ by

$$
w^{\alpha}(S)= \begin{cases}\alpha v(S) & \text { if } S \subset N \\ v(N) & \text { if } S=N\end{cases}
$$

\footnotetext{
1 Efficient solutions are often called values.
} 
Then,

$$
\varphi^{\alpha}(N, v)=C I S\left(N, w^{\alpha}\right) \text { for all } \alpha \in[0,1] \text { and }(N, v) \in \mathcal{G} \text {. }
$$

Next we state some well-known properties of solutions for TU-games. Players $i, j \in N$ are symmetric in game $(N, v)$ if $v(S \cup\{i\})=v(S \cup\{j\})$ for all $S \subseteq N \backslash\{i, j\}$. Player $i \in N$ is a null player in game $(N, v)$ if $v(S \cup\{i\})=v(S)$ for all $S \subseteq N \backslash\{i\}$. For a game $(N, v) \in \mathcal{G}$ and a permutation $\pi: N \rightarrow N$, the permuted game $(N, \pi v)$ is defined by $\pi v(S)=v(\{\pi(i) \mid i \in S\})$ for all $S \subseteq N$. For games $(N, v),(N, w) \in \mathcal{G}$ and $a, b \in \mathbb{R}$, the game $(N, a v+b w) \in \mathcal{G}$ is defined by $(a v+b w)(S)=a v(S)+b w(S)$ for all $S \subseteq N$. A game $(N, v) \in \mathcal{G}$ is nonnegative if $v(S) \geq 0$ for all $S \subseteq N$. Finally, a game $(N, v) \in \mathcal{G}$ is weakly essential if $\sum_{i \in N} v(\{i\}) \leq v(N)$. A solution $\psi$

- satisfies efficiency on $\mathcal{C} \subseteq \mathcal{G}$ if $\sum_{i \in N} \psi_{i}(N, v)=v(N)$ for all $(N, v) \in \mathcal{C}$;

- satisfies symmetry on $\mathcal{C} \subseteq \mathcal{G}$ if $\psi_{i}(N, v)=\psi_{j}(N, v)$ whenever $i$ and $j$ are symmetric players in $(N, v)$ for all $(N, v) \in \mathcal{C}$;

- satisfies linearity on $\mathcal{C} \subseteq \mathcal{G}$ if $\psi(N, a v+b w)=a \psi(N, v)+b \psi(N, w)$ for all $(N, v),(N, w) \in \mathcal{C}$ and all $a, b \in \mathbb{R}$ such that $(N, a v+b w) \in \mathcal{C}$;

- satisfies covariance on $\mathcal{C} \subseteq \mathcal{G}$ if for every pair of games $(N, v),(N, w) \in \mathcal{C}$ such that there is $a>0$ and $b \in \mathbb{R}$ with $w(S)=a v(S)+\sum_{i \in S} b_{i}$ for all $S \subseteq N$, it holds that $\psi_{i}(N, w)=a \psi_{i}(N, v)+b_{i}$ for all $i \in N$.

- satisfies individual rationality on $\mathcal{C} \subseteq \mathcal{G}$ if $\psi_{i}(N, v) \geq v(\{i\})$ for all $i \in N$ and all weakly essential games $(N, v) \in \mathcal{C}$;

- satisfies nonnegativity on $\mathcal{C} \subseteq \mathcal{G}$ if $\psi_{i}(N, v) \geq 0$ for all $i \in N$ and all nonnegative, weakly essential $(N, v) \in \mathcal{C}$;

- satisfies local monotonicity on $\mathcal{C} \subseteq \mathcal{G}$ if $\psi_{i}(N, v) \geq \psi_{j}(N, v)$ whenever $v(S \cup$ $\{i\}) \geq v(S \cup\{j\})$ for all $i, j \in N$, all $S \subseteq N \backslash\{i, j\}$, and all $(N, v) \in \mathcal{C}$;

- satisfies $\alpha$-standardness for two-player games on $\mathcal{C} \subseteq \mathcal{G}$ if for every $(N, v) \in \mathcal{C}$ with $N=\{i, j\}, i \neq j$, it holds that

$$
\psi_{i}(N, v)=\alpha v(\{i\})+\frac{1}{2}[v(N)-\alpha(v(\{i\})+v(\{j\}))] .
$$

The first three axioms are standard axioms often required for cooperative game solutions. Individual rationality and nonnegativity are two different axioms that give a lower bound to the payoffs of players. Relating to our previous remark on what right players have on their singleton worth, individual rationality expresses that players have full right on their singleton worths if the total worth of the grand coalition allows this. On the other hand, even if players cannot claim their singleton worth, nonnegativity requires that in any case no player has to pay if the worth of the grand coalition is large enough in the sense that all singleton worths can be paid. Local monotonicity is used in Levinský and Silársky (2004) and is also known as desirability, see Peleg and Sudhölter (2003). It expresses that a player, who contributes to every coalition at least as much as another player, should earn at least as much as that other player. The last property is used by Joosten (1996) to characterize the class of $\alpha$-egalitarian Shapley values. Standardness for two players games is obtained for $\alpha=1$, and egalitarian standardness for $\alpha=0$. 


\section{Characterizations for two-player games}

In the following sections we use $\alpha$-standardness to characterize solutions in the class $\Phi$. In order to have axiomatizations on the class $\Phi$ with no parameterized axiom, in this section we first support $\alpha$-standardness by showing how $\alpha$-standardness can be characterized on the class of two-player games by axioms that do not depend on $\alpha$. We denote the class of all two-player TU-games by $\mathcal{G}^{2}$.

In van den Brink and Funaki (2009, Proposition 4.2) it is shown that any solution that satisfies efficiency, symmetry, and linearity on the class of two-player games also satisfies $\alpha$-standardness for some $\alpha \in[0,1]$. Since we do not need linearity in the following sections, in this section we characterize $\alpha$-standardness for twoplayer games without linearity. Note that on the class of two-player games, a solution satisfying $\alpha$-standardness for some $\alpha \in[0,1]$ is equivalent to saying that the solution belongs to $\Phi$.

First, we impose an axiom stating that changing only the worth of the 'grand coalition' changes the payoffs of all players by the same amount. This is a weakening of fairness ${ }^{2}$ as used by van den Brink (2001) in axiomatizing the Shapley value.

Axiom 3.1 A solution $\psi$ satisfies weak fairness on $\mathcal{C} \subseteq \mathcal{G}$ if for every pair of games $(N, v),(N, w) \in \mathcal{C}$ such that $v(S)=w(S)$ for all $S \subset N$, there exists $c^{*} \in \mathbb{R}$ such that $\psi_{i}(N, v)-\psi_{i}(N, w)=c^{*}$ for all $i \in N$.

Adding homogeneity and a weak covariance property characterizes the class of solutions $\Phi$, i.e., $\alpha$-standardness for two-player games.

Axiom 3.2 A solution $\psi$ satisfies homogeneity (of degree 1) on $\mathcal{C} \subseteq \mathcal{G}$ if for every game $(N, v) \in \mathcal{C}$ and $c \in \mathbb{R}$ such that $(N, c v) \in \mathcal{C}$, it holds that $\psi(N, c v)=c \psi(N, v)$.

Axiom 3.3 A solution $\psi$ satisfies weak covariance on $\mathcal{C} \subseteq \mathcal{G}$ if for every pair of games $(N, v),(N, w) \in \mathcal{C}$ such that there is $c \in \mathbb{R}$ with $w(S)=v(S)+|S| c$ for all $S \subseteq N$, it holds that $\psi_{i}(N, w)=\psi_{i}(N, v)+c$ for all $i \in N$.

Note that both axioms are weaker than covariance. ${ }^{3}$

We prove the characterization of $\alpha$-standardness for two-player games by a series of lemmas. We first fix the player set $N=\{1,2\}$. A game $(N, v) \in \mathcal{G}$ is inessential if $\sum_{i \in N} v(\{i\})=v(N)$. In the following, let $\mathcal{G}_{A}^{\{1,2\}}$ be the class of all inessential (additive) games on $N=\{1,2\}$, and let $\mathcal{G}_{1_{0}}^{\{1,2\}}$ be the class of all inessential games on $N=\{1,2\}$ such that $v(\{1\})=0($ and thus $v(\{2\})=v(N))$.

Lemma 3.4 A solution $\psi$ on $\mathcal{G}_{1_{0}}^{\{1,2\}}$ satisfies efficiency, homogeneity, nonnegativity, and local monotonicity if and only if for some $\alpha \in[0,1]$, $\psi$ satisfies $\alpha$-standardness on $\mathcal{G}_{1_{0}}^{\{1,2\}}$.

\footnotetext{
${ }^{2}$ A solution $\psi$ satisfies fairness on $\mathcal{G}$ if $\psi_{i}(N, v+z)-\psi_{i}(N, v)=\psi_{j}(N, v+z)-\psi_{j}(N, v)$ whenever $i$ and $j$ are symmetric players in $(N, z)$.

${ }^{3}$ Note that weak covariance is also implied by efficiency and fairness (see Footnote 2) together since all players are symmetric in the game $(N, z)$ with $z(S)=|S| c$ for all $S \subseteq N$ and $w=v+z$.
} 
Proof It is obvious that each $\varphi^{\alpha} \in \Phi$ satisfies efficiency, homogeneity, nonnegativity, and local monotonicity on $\mathcal{G}_{1_{0}}^{\{1,2\}}$. Conversely, let $\psi$ be a solution satisfying the four axioms. Let $(N, v) \in \mathcal{G}_{1_{0}}^{\{1,2\}}$ such that $v(\{2\})=1$. By nonnegativity, $\psi_{1}(N, v) \geq 0$. By efficiency and local monotonicity, $\psi_{2}(N, v) \geq \frac{1}{2}$. By efficiency, there exists an $\alpha \in[0,1]$ such that $\psi_{1}(N, v)=\frac{1-\alpha}{2}$ and $\psi_{2}(N, v)=\frac{\alpha+1}{2}$. For any $(N, w) \in \mathcal{G}_{1_{0}}^{\{1,2\}}$, since there exists $c=w(\{2\}) \in \mathbb{R}$ such that $\psi(N, w)=\psi(N, c v)$, by homogeneity, we have $\psi(N, w)=c \psi(N, v)=\left(\frac{w(\{2\})(1-\alpha)}{2}, \frac{w(\{2\})(\alpha+1)}{2}\right)=\varphi^{\alpha}(N, w)$, as desired.

Obviously, the same holds for the class of two-player inessential games $(\{1,2\}, v)$ with $v(\{2\})=0$.

Without homogeneity, we can prove that the solution assigns to every game a convex combination of the equal division solution and the CIS-value, but the solution does need to belong to the class $\Phi$ since the weights given to the equal division solution and the CIS-value need not be the same for different games.

Next, we show that adding weak covariance characterizes the $\alpha$-standard solutions on the class of all inessential (additive) games on $N=\{1,2\}$.

Lemma 3.5 A solution $\psi$ on $\mathcal{G}_{A}^{\{1,2\}}$ satisfies efficiency, homogeneity, nonnegativity, local monotonicity, and weak covariance if and only iffor some $\alpha \in[0,1], \psi$ satisfies $\alpha$-standardness on $\mathcal{G}_{A}^{\{1,2\}}$.

Proof It is obvious that solution $\varphi^{\alpha}$ satisfies efficiency, homogeneity, nonnegativity, local monotonicity, and weak covariance on $\mathcal{G}_{A}^{\{1,2\}}$. Conversely, let $\psi$ be a solution satisfying the five axioms, and $(N, v) \in \mathcal{G}_{A}^{\{1,2\}}$. Consider a game $(N, w)$ given by $w(\{i\})=v(\{i\})-v(\{1\})$ for $i \in\{1,2\}$, and $w(N)=v(N)-2 v(\{1\})$. By Lemma 3.4, $\psi(N, w)=\varphi^{\alpha}(N, w)$ for some fixed $\alpha \in[0,1]$. But then weak covariance implies that $\psi_{i}(N, v)=\psi_{i}(N, w)+v(\{1\})=\varphi_{i}^{\alpha}(N, w)+v(\{1\})=\varphi_{i}^{\alpha}(N, v)$ for $i \in\{1,2\}$.

Since $N$ is the only coalition with more than one player, adding weak fairness implies a characterization of $\alpha$-standardness for all games on $N=\{1,2\}$. $^{4}$

Theorem 3.6 A solution $\psi$ on $\mathcal{G}^{\{1,2\}}$ satisfies efficiency, homogeneity, nonnegativity, local monotonicity, weak covariance, and weak fairness if and only if for some $\alpha \in$ $[0,1], \psi$ satisfies $\alpha$-standardness on $\mathcal{G}^{\{1,2\}}$.

Next we show logical independence of the six axioms in Theorem 3.6 by the following six alternative solutions that do not satisfy $\alpha$-standardness for any $\alpha \in[0,1]$ :

1. The solution $\psi$ given by $\psi_{i}(N, v)=v(\{i\})$ for all $i \in N$ and $(N, v) \in \mathcal{G}^{\{1,2\}}$ satisfies the axioms of Theorem 3.6 except efficiency.

\footnotetext{
${ }^{4}$ In Theorem 3.6 and the preceding lemmas we can replace nonnegativity and local monotonicity by a new axiom called weak individual rationality stating that for every weakly essential game $(N, v) \in \mathcal{C}$ and all $i \in N$ it holds that $\psi_{i}(N, v) \geq \min \left\{v(\{i\}), \frac{v(N)}{|N|}\right\}$.
} 
2. The solution $\psi$ given by $\psi(N, v)=\operatorname{ED}(N, v)$ if $|v(\{1\})-v(\{2\})| \leq 10$, and $\psi(N, v)=\operatorname{CIS}(N, v)$ if $|v(\{1\})-v(\{2\})|>10$, satisfies the axioms of Theorem 3.6 except homogeneity.

3. The solution $\psi$ given by $\psi_{i}(N, v)=2 v(\{i\})+\frac{v(N)-\sum_{j \in N} 2 v(\{j\})}{|N|}$ for all $i \in N$ and $(N, v) \in \mathcal{G}^{\{1,2\}}$ satisfies the axioms of Theorem 3.6 except nonnegativity.

4. The solution $\psi$ given by

$$
\psi_{i}(N, v)=C I S_{j}(N, v) \quad \text { for all }(N, v) \in \mathcal{G}^{\{1,2\}}, \quad i \neq j,
$$

satisfies the axioms of Theorem 3.6 except local monotonicity.

5. The solution $\psi$ given by $\psi(N, v)=\operatorname{ED}(N, v)$ if $\frac{v(\{1\})}{v(\{2\})} \leq 10$, and $\psi(N, v)=$ $\operatorname{CIS}(N, v)$ if $\frac{v(\{1\})}{v(\{2\})}>10$ satisfies the axioms of Theorem 3.6 except weak covariance.

6. The solution $\psi$ given by

$$
\psi_{i}(N, v)= \begin{cases}v(\{i\})+\frac{v(N)-v(\{1\})-v(\{\{\})}{3} & \text { if } v(\{i\})<v(\{j\}), j \neq i \\ v(\{i\})+\frac{2(v(N)-v(\{1\})-v(\{2\}))}{3} & \text { if } v(\{i\})>v(\{j\}), j \neq i \\ \frac{v(N)}{2} & \text { if } v(\{1\})=v(\{2\}),\end{cases}
$$

for all $(N, v) \in \mathcal{G}^{\{1,2\}}$, satisfies the axioms of Theorem 3.6 except weak fairness.

Note that as corollaries from Theorem 3.6 we obtain characterizations of the equal division solution and the CIS-value, which extend to $n$-player games with the properties of consistency and population solidarity which are discussed in the next sections. As shown before, the equal division solution is the only solution in the class $\Phi$ that satisfies nonnegativity, and the CIS-value is the only solution in $\Phi$ that satisfies covariance or individual rationality. With Theorem 3.6 this immediately yields axiomatizations of the equal division solution and the CIS-value as corollaries. Further, it is obvious that adding anonymity characterizes $\alpha$-standardness on the class of all two-player games.

Next, we characterize specific solutions $\varphi^{\alpha}$ from the class $\Phi$ using a parameterized axiom that has nonnegativity and individual rationality as special cases. For $\alpha \in[0,1]$ we call a game $\alpha$-essential if $\sum_{i \in N} \alpha v(\{i\}) \leq v(N)$. Clearly, for $\alpha=0$ this boils down to $v(N) \geq 0$, while for $\alpha=1$ this is weak essentiality.

Axiom 3.7 Let $\alpha \in[0,1]$. A solution $\psi$ satisfies $\alpha$-individual rationality on $\mathcal{C} \subseteq \mathcal{G}$ if for every $\alpha$-essential game $(N, v) \in \mathcal{C}$ it holds that $\psi_{i}(N, v) \geq \alpha v(\{i\})$ for all $i \in N$.

Clearly $\alpha=1$ yields individual rationality, while $\alpha=0$ yields nonnegativity.

Earlier, we introduced weak fairness and gave a characterization of the class of solutions $\Phi$ for two-player games using axioms that do not depend on $\alpha$ in Theorem 3.6. For a specific $\alpha \in[0,1], \alpha$-standardness for two-player games is characterized by efficiency, weak fairness, and the corresponding $\alpha$-individual rationality.

Theorem 3.8 Let $\alpha \in[0,1]$. A solution $\psi$ on $\mathcal{G}^{2}$ satisfies efficiency, weak fairness, and $\alpha$-individual rationality if and only if it satisfies $\alpha$-standardness for two-player games. 
Proof It is obvious that on $\mathcal{G}^{2}, \varphi^{\alpha}$ satisfies efficiency, weak fairness, and $\alpha$-individual rationality. Conversely, let $\psi$ be a solution satisfying efficiency, weak fairness, and $\alpha$-individual rationality for some $\alpha \in[0,1]$. Let $(N, v)$ be a two-player game with $N=\{i, j\}, i \neq j$. First, consider a game $(N, w)$ given by $w(\{i\})=v(\{i\}), w(\{j\})=$ $v(\{j\})$, and $w(N)=\alpha(v(\{i\})+v(\{j\}))$. Since $(N, w)$ is an $\alpha$-essential game, $\alpha$ individual rationality implies that $\psi_{i}(N, w) \geq \alpha w(\{i\})=\alpha v(\{i\})$ and $\psi_{j}(N, w) \geq$ $\alpha w(\{j\})=\alpha v(\{j\})$. Efficiency then implies that these inequalities are equalities. But then weak fairness implies that $\psi_{i}(N, v)-\alpha v(\{i\})=\psi_{j}(N, v)-\alpha v(\{j\})$. With efficiency it follows that $\psi(N, v)=\varphi^{\alpha}(N, v)$.

Taking $\alpha=0$ and $\alpha=1$ we obtain the following corollaries. ${ }^{5}$

Corollary 3.9 (i) A solution $\psi$ satisfies efficiency, weakfairness, and nonnegativity if and only if it satisfies egalitarian standardness for two-player games.

(ii) A solution $\psi$ satisfies efficiency, weak fairness, and individual rationality if and only if it satisfies standardness for two-player games.

\section{Consistency and egalitarian solutions}

In this section we consider the extension of the solutions in the previous section to $n$-player games. We can use the reduced game introduced by van den Brink and Funaki (2009, Section 5), but since we consider only convex combinations of the equal division solution and the CIS-value, we can make a simplification of their reduced game.

Take a game $(N, v) \in \mathcal{G}$, a payoff vector $x \in \mathbb{R}^{N}$ and a player $j \in N$. The player set of the reduced game is obtained by removing player $j$ from the original player set $N$. The worths of the coalitions in this reduced game reflect what these coalitions can earn if player $j$ has left the game with its payoff $x_{j}$. The worth of the coalition $N \backslash\{j\}$ (the 'grand coalition') in the reduced game is equal to the worth of $N$ minus the payoff $x_{j}$ assigned to player $j$. Clearly, this is what is left to be allocated to the players in $N \backslash\{j\}$ after removing player $j$ from the game with payoff $x_{j}$. For the other coalitions $S \subset N \backslash\{j\}$ we consider the projection reduced game (see, e.g., Oishi et al. 2016; Funaki and Yamato 2001) where it is assumed that coalition $S$ simply earns its worth $v(S)$ in the original game. ${ }^{6}$

Definition 4.1 Given a game $(N, v) \in \mathcal{G}$ with $|N| \geq 3$, a player $j \in N$ and a payoff vector $x \in \mathbb{R}^{N}$, the projection reduced game with respect to $j$ and $x$ is the game $\left(N \backslash\{j\}, v^{x}\right)$ given by

$$
v^{x}(S)= \begin{cases}v(N)-x_{j} & \text { if } S=N \backslash\{j\} \\ v(S) & \text { if } S \subset N \backslash\{j\} .\end{cases}
$$

\footnotetext{
5 Recall that van den Brink and Funaki (2009) characterized the equal division solution (respectively CISvalue) by efficiency, symmetry, linearity, and a weaker nonnegativity requiring nonnegative payoffs only if the worths of all coalitions are nonnegative (respectively, individual rationality).

6 In van den Brink and Funaki (2009) it is assumed that in the reduced game a coalition has the participation of the leaving player $j$ (but must pay $x_{j}$ to $j$ ) or not. Also, because of the simplification we do not have to consider the case $|N|=3$ differently from the case $|N| \geq 4$, as done in van den Brink and Funaki (2009).
} 
Although we can allow for different worths for coalitions of size strictly between 1 and $|N|-1$, we consider only the (projection) game given above. ${ }^{7}$ The corresponding consistency property of a solution is the following: ${ }^{8}$

Definition 4.2 A solution $\psi$ satisfies projection consistency if and only if for every $(N, v) \in \mathcal{G}$ with $|N| \geq 3, j \in N$, and $x=\psi(N, v)$ it holds that $\psi_{i}\left(N \backslash\{j\}, v^{x}\right)=$ $\psi_{i}(N, v)$ for all $i \in N \backslash\{j\}$.

Projection consistency implies that given a game $(N, v)$, if $x$ is a solution payoff vector for $(N, v)$, then for every player $j \in N$, the payoff vector $x_{N \backslash\{j\}}$ with payoffs for the players in $N \backslash\{j\}$, must be the solution payoff vector of the projection reduced game $\left(N \backslash\{j\}, v^{x}\right)$. It is a kind of internal consistency requirement to guarantee that players respect the recommendations made by the solution.

Proposition 4.3 For every $\alpha \in[0,1]$ the solution $\varphi^{\alpha}$ satisfies projection consistency on the class of all games $\mathcal{G}$.

Proof ${ }^{9}$ Take any $\alpha \in[0,1]$, and any $(N, v) \in \mathcal{G}$ with $|N| \geq 3$. For $x=\varphi^{\alpha}(N, v)$ and $i \in N \backslash\{j\}$, we have

$$
\begin{aligned}
\varphi_{i}^{\alpha}\left(N \backslash\{j\}, v^{x}\right)= & \alpha v^{x}(\{i\})+\frac{1}{|N|-1}\left(v^{x}(N \backslash\{j\})-\sum_{k \in N \backslash\{j\}} \alpha v^{x}(\{k\})\right) \\
= & \alpha v(\{i\})+\frac{1}{|N|-1}\left(v(N)-x_{j}-\sum_{k \in N \backslash\{j\}} \alpha v(\{k\})\right) \\
= & \alpha v(\{i\})+\frac{1}{|N|-1}(v(N)-\alpha v(\{j\}) \\
& \left.-\frac{1}{|N|}\left(v(N)-\sum_{k \in N} \alpha v(\{k\})\right)-\sum_{k \in N \backslash\{j\}} \alpha v(\{k\})\right) \\
= & \alpha v(\{i\})+\frac{1}{|N|-1} \cdot \frac{|N|-1}{|N|} \cdot\left(v(N)-\sum_{k \in N} \alpha v(\{k\})\right) \\
= & \varphi_{i}^{\alpha}(N, v) .
\end{aligned}
$$

\footnotetext{
${ }^{7}$ Here we only consider the class $\mathcal{G}$ of all TU-games. If one considers subclasses $\mathcal{C} \subset \mathcal{G}$, then in the definition of consistency one should additionally require that the reduced game $\left(N \backslash\{j\}, v^{x}\right)$ in this definition also belongs to $\mathcal{C}$.

8 The equal division solution satisfies several well-known consistency axioms that are also satisfied by the Shapley value (Shapley 1953) such as those of Sobolev (1973) and Hart and Mas-Colell (1988, 1989), which are not satisfied by the CIS-value. In van den Brink et al. (2013) it is shown that all convex combinations of the Shapley value and equal division solution as introduced in Joosten (1996), satisfy Sobolev (1973)'s consistency. Ju et al. (2007) consider the convex combinations of the Shapley value and the CIS-value.

9 Since we slightly changed the reduced game of van den Brink and Funaki (2009) for $\beta=1$, we give the short proof, which follows similar lines as that of their Proposition 5.3. Their case $|N| \geq 4$ with $\beta=1$ now also holds for the case $|N|=3$.
} 
Adding $\alpha$-standardness for two-player games characterizes ${ }^{10}$ the solution $\varphi^{\alpha}$.

Theorem 4.4 Let $\alpha \in[0,1]$. A solution $\psi$ satisfies $\alpha$-standardness for two-player games and projection consistency on the class of all games $\mathcal{G}$ if and only if $\psi=\varphi^{\alpha}$.

Proof The solution $\varphi^{\alpha}$ satisfying $\alpha$-standardness for two-player games is straightforward. Projection consistency follows from Proposition 4.3. Here we prove the 'only if' part. Take $\alpha \in[0,1]$, and let $\psi$ be a solution which satisfies $\alpha$-standardness for two-player games and projection consistency. If $|N|=2$, then $\psi(N, v)=\varphi^{\alpha}(N, v)$ follows from $\alpha$-standardness for two-player games.

Proceeding by induction, for $|N| \geq 3$, suppose that $\psi\left(N^{\prime}, v^{\prime}\right)=\varphi^{\alpha}\left(N^{\prime}, v^{\prime}\right)$ whenever $\left|N^{\prime}\right|=|N|-1$. We will show that $\psi(N, v)=\varphi^{\alpha}(N, v)$. Let $x=\psi(N, v)$ and $y=\varphi^{\alpha}(N, v)$. Let $j \in N$ be such that $\left|x_{j}-y_{j}\right|=\min \left\{\left|x_{k}-y_{k}\right| \mid k \in N\right\}$. Take any $i \in N \backslash\{j\}$. For the two reduced games $\left(N \backslash\{j\}, v^{x}\right)$ and $\left(N \backslash\{j\}, v^{y}\right)$, by projection consistency of $\varphi^{\alpha}$ and $\psi$, and the induction hypothesis, we have

$$
x_{i}-y_{i}=\psi_{i}\left(N \backslash\{j\}, v^{x}\right)-\varphi_{i}^{\alpha}\left(N \backslash\{j\}, v^{y}\right)=\varphi_{i}^{\alpha}\left(N \backslash\{j\}, v^{x}\right)-\varphi_{i}^{\alpha}\left(N \backslash\{j\}, v^{y}\right) .
$$

By definition of $\varphi^{\alpha}$ and the projection reduced game, we have

$$
\begin{aligned}
\varphi_{i}^{\alpha}\left(N \backslash\{j\}, v^{x}\right)-\varphi_{i}^{\alpha}\left(N \backslash\{j\}, v^{y}\right) \\
=\alpha v^{x}(\{i\})+\frac{1}{|N|-1}\left(v(N \backslash\{j\})-x_{j}-\sum_{k \in N \backslash\{j\}} \alpha v^{x}(\{k\})\right)-\alpha v^{y}(\{i\}) \\
\quad-\frac{1}{|N|-1}\left(v(N \backslash\{j\})-y_{j}-\sum_{k \in N \backslash\{j\}} \alpha v^{y}(\{k\})\right) \\
=\alpha v(\{i\})-\alpha v(\{i\})-\frac{1}{|N|-1} \sum_{k \in N \backslash\{j\}}(\alpha v(\{k\})-\alpha v(\{k\}))+\frac{1}{|N|-1}\left(-x_{j}+y_{j}\right) \\
=\frac{1}{|N|-1}\left(-x_{j}+y_{j}\right) .
\end{aligned}
$$

With (4.1) this implies that $\left|x_{i}-y_{i}\right|=\frac{1}{|N|-1}\left|x_{j}-y_{j}\right| \leq \frac{1}{2}\left|x_{j}-y_{j}\right|$ since $|N| \geq 3$. From the choice of $j$, it follows that $\left|x_{j}-y_{j}\right| \leq\left|x_{i}-y_{i}\right| \leq \frac{1}{2}\left|x_{j}-y_{j}\right|$, which implies that $x_{i}-y_{i}=0$ and also $x_{j}-y_{j}=0$. Therefore, $\psi(N, v)=\varphi^{\alpha}(N, v)$. This completes the proof.

By Theorems 3.8 and 4.4, we obtain the following corollary:

Corollary 4.5 Let $\alpha \in[0,1]$. A solution $\psi$ satisfies efficiency, weak fairness, $\alpha$ individual rationality, and projection consistency on the class of all games $\mathcal{G}$ if and only if $\psi=\varphi^{\alpha}$.

\footnotetext{
10 Note that, compared to van den Brink and Funaki (2009), we do not need efficiency.
} 


\section{Population solidarity and egalitarian solutions}

Now we consider another extension of $\alpha$-standardness to $n$-player games by imposing the axiom of population solidarity. Population solidarity requires that upon the arrival of a new player all the original players should be affected in the same direction, all weakly gain or all weakly lose. Its implications have been studied in various contexts (Thomson 1983; Chun 1986), and for TU-games by Chun and Park (2012).

Axiom 5.1 A solution $\psi$ satisfies population solidarity if for all $(N, v),\left(N^{\prime}, w\right) \in \mathcal{G}$ satisfying $N \subset N^{\prime}$ and $v(S)=w(S)$ for all $S \subseteq N$, it holds that either $\psi_{j}(N, v) \geq$ $\psi_{j}\left(N^{\prime}, w\right)$ for all $j \in N$, or $\psi_{j}(N, v) \leq \psi_{j}\left(N^{\prime}, w\right)$ for all $j \in N$.

It is easy to check that the convex combinations of the equal division solution and the CIS-value are the only ones in the class of equal surplus division solutions considered in van den Brink and Funaki (2009) that satisfy population solidarity. Chun and Park (2012, Theorem 1) show that standardness for two-player games, efficiency and population solidarity characterize the CIS-value. In a similar way we can show the following theorem. The proof is essentially the same as that in Chun and Park (2012) and can be found in the appendix.

Theorem 5.2 Let $\alpha \in[0,1]$. A solution $\psi$ satisfies efficiency, $\alpha$-standardness for two-player games and population solidarity if and only if $\psi=\varphi^{\alpha}$.

Logical independence of the axioms in Theorem 5.2 follows from the following three alternative solutions:

1. The solution $\psi^{\alpha}$, given by $\psi_{i}^{\alpha}(N, v)=\alpha v(\{i\})+\frac{1}{2} \sum_{\substack{T \subseteq N \\|T|=2}}\left(v(T)-\alpha \sum_{k \in T}\right.$ $v(\{k\}))$ for all $(N, v) \in \mathcal{G}$ and $i \in N$, satisfies $\alpha$-standardness for two-player games and population solidarity. It does not satisfy efficiency.

2. For all $i \in \mathbb{N}$, let $t_{i}$ be a number assigned to player $i$, such that these numbers are distinct. The solution $\psi$, for all $(N, v) \in \mathcal{G}$ given by $\psi(N, v)=\varphi^{\alpha}(N, w)$, where $(N, w)$ is a game such that $w(\{i\})=t_{i} v(\{i\})$ for all $i \in N$ and $w(S)=v(S)$ for any other $S$, satisfies efficiency and population solidarity. It does not satisfy $\alpha$-standardness for two-player games.

3. The egalitarian Shapley values satisfy efficiency and $\alpha$-standardness for two-player games. It does not satisfy population solidarity.

By Theorems 3.8 and 5.2, we obtain the following corollary:

Corollary 5.3 Let $\alpha \in[0,1]$. A solution $\psi$ satisfies efficiency, weak fairness, $\alpha$ individual rationality, and population solidarity if and only if $\psi=\varphi^{\alpha}$.

Theorem 5.2 characterizes each solution $\varphi^{\alpha}, \alpha \in[0,1]$. It is an immediate consequence of Theorems 3.6 and 5.2 that adding anonymity characterizes all solutions on $\mathcal{G}$, that is a solution $\psi$ on $\mathcal{G}$ belongs to $\Phi$ if and only if it satisfies efficiency, homogeneity, local monotonicity, nonnegativity, weak covariance, weak fairness, anonymity, and population solidarity. It turns out that we can even do without anonymity. To show this it is sufficient to prove that under efficiency and population solidarity, $\alpha$ does not depend on the choice of the player set. 
For any $(N, v) \in \mathcal{G}$ and $T \subset N$, the subgame $\left(T, v_{T}\right)$ is given by $v_{T}(S)=v(S)$ for all $S \subseteq T$.

Theorem 5.4 A solution $\psi$ on $\mathcal{G}$ belongs to $\Phi$ if and only if it satisfies efficiency, homogeneity, local monotonicity, nonnegativity, weak covariance, weak fairness, and population solidarity.

Proof It is clear that the 'only if' part is satisfied. To show the 'if' part, by Theorems 3.6 and 5.2 it is sufficient to prove that under efficiency and population solidarity, a solution that satisfies $\alpha$-standardness for games on a player set $N=\{i, j\}, i \neq j$, must satisfy the same $\alpha$-standardness on any class of games on player sets $N^{\prime} \subset \mathbb{N}$ with $\left|N^{\prime}\right|=2$. Let $\psi$ be a solution that satisfies efficiency, population solidarity, and $\alpha_{i j}$-standardness for two-player games on $\mathcal{G}^{\{i, j\}}$ for any $i, j \in \mathbb{N}$. We will show that $\alpha_{i j}=\alpha_{i^{\prime} j^{\prime}}$ for any $i, j, i^{\prime}, j^{\prime} \in \mathbb{N}$.

Suppose by contradiction that there exist $i, j, k \in \mathbb{N}$ such that $\alpha_{i j} \neq \alpha_{i k}$. For simplicity, we assume that $i=1, j=2, k=3$, and $\alpha_{12}>\alpha_{13}$. Let $\delta$ and $\epsilon$ be positive real numbers such that

$$
\begin{aligned}
& \alpha_{12}-\alpha_{13}<\delta<2\left(\alpha_{12}-\alpha_{13}\right) \\
& 0<\epsilon<2 \alpha_{12}-2 \alpha_{13}-\delta
\end{aligned}
$$

Then it follows that

$$
3 \alpha_{12}+2 \alpha_{13}+\delta<5 \alpha_{12}-\epsilon \text {. }
$$

Now, consider the following game $(N, v) \in \mathcal{G}$ where $N=\{1,2,3\}$ and $v$ is defined as follows:

\begin{tabular}{lllllll}
\hline$S$ & $\{1\}$ & $\{2\}$ & $\{3\}$ & $\{1,2\}$ & $\{2,3\}$ & $\{1,3\}$ \\
\hline$v(S)$ & 1 & 2 & 2 & $3 \alpha_{12}$ & $4 \alpha_{12}-2 \epsilon$ & $3 \alpha_{13}+2 \delta$ \\
\hline
\end{tabular}

and $v(N)$ is any real number satisfying

$$
3 \alpha_{12}+2 \alpha_{13}+\delta<v(N)<5 \alpha_{12}-\epsilon \text {. }
$$

Note that, by $\alpha_{i j}$-standardness for two-player games,

$$
\begin{aligned}
& \psi_{1}\left(\{1,2\}, v_{\{1,2\}}\right)=\alpha_{12}+\frac{1}{2}\left(3 \alpha_{12}-3 \alpha_{12}\right)=\alpha_{12} \\
& \psi_{2}\left(\{1,2\}, v_{\{1,2\}}\right)=2 \alpha_{12}+\frac{1}{2}\left(3 \alpha_{12}-3 \alpha_{12}\right)=2 \alpha_{12} \\
& \psi_{2}\left(\{2,3\}, v_{\{2,3\}}\right)=2 \alpha_{23}+\frac{1}{2}\left(4 \alpha_{12}-2 \epsilon-4 \alpha_{23}\right)=2 \alpha_{12}-\epsilon
\end{aligned}
$$




$$
\begin{aligned}
& \psi_{3}\left(\{2,3\}, v_{\{2,3\}}\right)=2 \alpha_{23}+\frac{1}{2}\left(4 \alpha_{12}-2 \epsilon-4 \alpha_{23}\right)=2 \alpha_{12}-\epsilon \\
& \psi_{1}\left(\{1,3\}, v_{\{1,3\}}\right)=\alpha_{13}+\frac{1}{2}\left(3 \alpha_{13}+2 \delta-3 \alpha_{13}\right)=\alpha_{13}+\delta \\
& \psi_{3}\left(\{1,3\}, v_{\{1,3\}}\right)=2 \alpha_{13}+\frac{1}{2}\left(3 \alpha_{13}+2 \delta-3 \alpha_{13}\right)=2 \alpha_{13}+\delta .
\end{aligned}
$$

We distinguish the following three cases:

1. Suppose that $\psi_{1}(N, v)<\alpha_{12}$. Applying population solidarity to $\left(\{1,2\}, v_{\{1,2\}}\right)$ and $(N, v)$ yields $\psi_{2}(N, v) \leq 2 \alpha_{12}$. Since $\alpha_{12}<\alpha_{13}+\delta$, it follows that $\psi_{1}(N, v)<\alpha_{13}+\delta$. Applying population solidarity to $\left(\{1,3\}, v_{\{1,3\}}\right)$ and $(N, v)$ yields $\psi_{3}(N, v) \leq 2 \alpha_{13}+\delta$. Then,

$$
\begin{aligned}
v(N) & =\psi_{1}(N, v)+\psi_{2}(N, v)+\psi_{3}(N, v)<\alpha_{12}+2 \alpha_{12}+2 \alpha_{13}+\delta \\
& =3 \alpha_{12}+2 \alpha_{13}+\delta,
\end{aligned}
$$

a contradiction to (5.1).

2. Suppose that $\psi_{1}(N, v)>\alpha_{12}$. Applying population solidarity to $\left(\{1,2\}, v_{\{1,2\}}\right)$ and $(N, v)$ yields $\psi_{2}(N, v) \geq 2 \alpha_{12}$. Since $\psi_{2}(N, v)>2 \alpha_{12}-\epsilon$, applying population solidarity to $\left(\{2,3\}, v_{\{2,3\}}\right)$ and $(N, v)$ yields $\psi_{3}(N, v) \geq 2 \alpha_{12}-\epsilon$. Then,

$$
\begin{aligned}
v(N) & =\psi_{1}(N, v)+\psi_{2}(N, v)+\psi_{3}(N, v)>\alpha_{12}+2 \alpha_{12}+2 \alpha_{12}-\epsilon \\
& =5 \alpha_{12}-\epsilon,
\end{aligned}
$$

a contradiction to (5.1).

3. Suppose that $\psi_{1}(N, v)=\alpha_{12}$. Then, $\psi_{1}(N, v)<\alpha_{13}+\delta$. Applying population solidarity to $\left(\{1,3\}, v_{\{1,3\}}\right)$ and $(N, v)$ yields $\psi_{3}(N, v) \leq 2 \alpha_{13}+\delta$. Since $2 \alpha_{13}+$ $\delta<2 \alpha_{12}-\epsilon$, it holds that $\psi_{3}(N, v)<2 \alpha_{12}-\epsilon$. Applying population solidarity to $\left(\{2,3\}, v_{\{2,3\}}\right)$ and $(N, v)$ yields $\psi_{2}(N, v) \leq 2 \alpha_{12}-\epsilon$. Then, since $\psi_{3}(N, v) \leq$ $2 \alpha_{13}+\delta$ and $\psi_{2}(N, v) \leq 2 \alpha_{12}-\epsilon$,

$$
\begin{aligned}
v(N) & =\psi_{1}(N, v)+\psi_{2}(N, v)+\psi_{3}(N, v) \leq \alpha_{12}+2 \alpha_{12}-\epsilon+2 \alpha_{13}+\delta \\
& <3 \alpha_{12}+2 \alpha_{13}+\delta
\end{aligned}
$$

a contradiction to (5.1).

We note that in this theorem, homogeneity, local monotonicity, nonnegativity, weak covariance, and weak fairness can be replaced by symmetry and linearity.

\section{The ENSC-value}

The dual game $\left(N, v^{*}\right)$ of a game $(N, v)$ is the game that assigns to each coalition $S \subseteq N$ the worth that is lost by the 'grand coalition' $N$ if coalition $S$ leaves $N$, i.e.,

$$
v^{*}(S)=v(N)-v(N \backslash S) \text { for all } S \subseteq N
$$


The ENSC-value assigns to every game $(N, v)$ the CIS-value of its dual game, i.e., for all $i \in N$,

$$
\begin{aligned}
\operatorname{ENSC}_{i}(N, v) & =\operatorname{CIS}_{i}\left(N, v^{*}\right)=v^{*}(\{i\})+\frac{1}{|N|}\left(v^{*}(N)-\sum_{j \in N} v^{*}(\{j\})\right) \\
& =v(N)-v(N \backslash\{i\})+\frac{1}{|N|}\left(v(N)-\sum_{j \in N}(v(N)-v(N \backslash\{j\}))\right) \\
& =-v(N \backslash\{i\})+\frac{1}{|N|}\left(v(N)+\sum_{j \in N} v(N \backslash\{j\})\right) .
\end{aligned}
$$

Thus, the ENSC-value assigns to every player in a game its marginal contribution to the 'grand coalition' and distributes the (positive or negative) remainder equally among the players.

It is known that the ENSC-value is the dual of the CIS-value and has several dual properties, see, e.g., Funaki (1998) and Oishi et al. (2016). In this section we present two properties for values that are defined as a convex combination of the ENSC-value and the equal division solution. These properties are induced from the theorems in the previous two sections by their duality. Thus we omit the proofs of the two theorems. Note that for two-player games, both solutions coincide (with any standard solution).

For each $\alpha \in[0,1]$, let $\bar{\varphi}^{\alpha}$, for all $(N, v) \in \mathcal{G}$, be given by

$$
\bar{\varphi}^{\alpha}(N, v)=\alpha \operatorname{ENSC}(N, v)+(1-\alpha) \operatorname{ED}(N, v) .
$$

It is easy to check that the solution $\bar{\varphi}^{\alpha}$ is the dual of $\varphi^{\alpha}$, that is, $\bar{\varphi}^{\alpha}(N, v)=\varphi^{\alpha}\left(N, v^{*}\right)$ $(N, v) \in \mathcal{G}$.

From Oishi et al. $(2012,2016)$ it follows that, for any game $(N, v) \in \mathcal{G}$ with $|N| \geq 3$, a player $j \in N$, and a payoff vector $x \in \mathbb{R}^{N}$, the dual of the projection reduced game defined in Definition 4.2 (we call this the dual projection reduced game with respect to $j$ and $x)$ is the game $\left(N \backslash\{j\},\left(v^{x}\right)^{*}\right)$ given by

$$
\left(v^{x}\right)^{*}(S)= \begin{cases}v(S \cup\{j\})-x_{j} & \text { if } S \subseteq N \backslash\{j\}, S \neq \emptyset \\ 0 & \text { if } S=\emptyset\end{cases}
$$

The consistency property related to this reduced game is called dual projection consistency and is defined similarly as Definition 4.2. Together with $\alpha$-standardness for two-player games it characterizes the corresponding convex combination of the equal division solution and the CIS-value.

Theorem 6.1 Let $\alpha \in[0,1]$. A solution $\phi$ satisfies $\alpha$-standardness for two-player games and dual projection consistency on the class of all games $\mathcal{G}$ if and only if $\phi=\bar{\varphi}^{\alpha}$. 
Now we consider the dual property of population solidarity. It is obtained by replac$\operatorname{ing}(N, v)$ by $\left(N, v^{*}\right)$ in the original property. For a game $\left(N^{\prime}, w\right)$ and $N \subset N^{\prime}$, let $\bar{w}(S)=w\left(S \cup\left(N^{\prime} \backslash N\right)\right)$ for all $S \subseteq N$.

Axiom 6.2 A solution $\psi$ satisfies the dual of population solidarity if for all $(N, v),\left(N^{\prime}, w\right) \in \mathcal{G}$ satisfying $N \subset N^{\prime}$ and $v(N)-v(N \backslash S)=\bar{w}(N)-\bar{w}(N \backslash S)$ for all $S \subseteq N$, it holds that either $\psi_{j}(N, v) \geq \psi_{j}\left(N^{\prime}, w\right)$ for all $j \in N$, or $\psi_{j}(N, v) \leq \psi_{j}\left(N^{\prime}, w\right)$ for all $j \in N$.

The dual of population solidarity requires the following: Consider two games $(N, v)$ and $\left(N^{\prime}, w\right)$ such that $N \subset N^{\prime}$. We compare the worth of the coalitions in the two games for the player set $N$. Then we consider $\bar{w}$ instead of $w$. If for any $S \subseteq N$, the contributions of $S$ to $N$ in both games coincide, all the original players in $N$ should be affected in the same direction.

Theorem 6.3 Let $\alpha \in[0,1]$. A solution $\phi$ satisfies efficiency, $\alpha$-standardness for two-player games and the dual of population solidarity if and only if $\phi=\bar{\varphi}^{\alpha}$.

Since $\bar{\varphi}^{\alpha}$ satisfies $\alpha$-standardness for two-player games, we can formulate a corollary similar to Corollary 4.5 .

\section{Concluding remarks}

In this paper, we showed that all convex combinations of the equal division solution and the CIS-value satisfy population solidarity, and we provided an axiomatization of the class of these solutions using population solidarity. For every specific solution in this class we provided an axiomatization using projection consistency and a paramatrized standardness axiom. Similar characterizations are obtained for convex combinations of the equal division solution and the ENSC-value.

A main goal of axiomatizing solutions is to compare different solutions based on their characterizing properties. Using the axiomatizations obtained in this paper we can (i) distinguish the class of solutions considered here from other solutions by the axiomatization using population solidarity (Theorem 5.4), and within this class distinguish each solution by its corresponding standardness for two-player games (Theorem 4.4).

One of the most applied solutions for TU-games is the Shapley value. In the literature there exist axiomatizations of the egalitarian Shapley values (being convex combinations of the Shapley value and the equal division solution, see, e.g., Joosten (1996) and van den Brink et al. (2013) and generalized consensus values (being convex combinations of the Shapley value and the equal surplus division solution (see Ju et al. 2007). A next question for future research is to obtain axiomatizations of convex combinations considered here and the Shapley value.

Open Access This article is distributed under the terms of the Creative Commons Attribution 4.0 International License (http://creativecommons.org/licenses/by/4.0/), which permits unrestricted use, distribution, and reproduction in any medium, provided you give appropriate credit to the original author(s) and the source, provide a link to the Creative Commons license, and indicate if changes were made. 


\section{Appendix: Proof of Theorem 5.2}

Here we present the proof of Theorem 5.2 which generalizes that of Chun and Park (2012) in a straightforward way. First, it is obvious that $\varphi^{\alpha}$ satisfies efficiency, $\alpha$ standardness for two-player games and population solidarity.

To prove uniqueness, let $\psi$ be a solution satisfying efficiency, $\alpha$-standardness for two-player games and population solidarity. We assume that $N=\{1, \ldots, n\}$. For any game $(N, v) \in \mathcal{G}$ and $\alpha \in[0,1]$, we define the real number $x^{\alpha}(N, v)$ by

$$
x^{\alpha}(N, v)=\frac{v(N)-\alpha \sum_{k \in N} v(\{k\})}{|N|},
$$

and we also define a vector $\theta^{\alpha}(N, v) \in \mathbb{R}^{N}$ by $\theta_{i}^{\alpha}(N, v)=\psi_{i}(N, v)-\alpha v(\{i\})$ for all $i \in N$. For notational convenience, in case there is no confusion we will often omit the superscript $\alpha$ and shortly write $x(N, v)$ and $\theta(N, v)$. Note that from efficiency,

$$
\sum_{i \in N} \theta_{i}(N, v)=\sum_{i \in N} \psi_{i}(N, v)-\alpha \sum_{i \in N} v(\{i\})=v(N)-\alpha \sum_{i \in N} v(\{i\})=x(N, v) \cdot|N| .
$$

If $|N|=2$, then $\psi(N, v)=\varphi^{\alpha}(N, v)$ follows from the assumption that $\psi$ satisfies $\alpha$-standardness for two-player games. Let $(N, v) \in \mathcal{G}$ with $|N| \geq 3$. For simplicity, let $N=\{1,2,3, \ldots, n\}$. It is sufficient to show that $\theta_{i}(N, v)=x(N, v)$ for all $i \in N$. We will show this by contradiction. Suppose that there is a player $k \in N$ such that $\theta_{k}(N, v) \neq x(N, v)$. Since $\sum_{i \in N} \theta_{i}(N, v)=x(N, v) \cdot|N|$, there exists a player $j \in N$ such that $\theta_{j}(N, v)>x(N, v)$. Without loss of generality, we may assume that $\theta_{1}(N, v)-x(N, v)>0$.

Let $\delta$ be a positive real number defined by

$$
\delta \equiv \frac{\theta_{1}(N, v)-x(N, v)}{2(n+1)^{2}} .
$$

Let $N^{\prime} \equiv\{1,2, \ldots, n+1\}$ and $N^{\prime \prime} \equiv\{1,2, \ldots, n+1, n+2\}$. We consider a game $\left(N^{\prime \prime}, w\right)$ such that for all $S \subset N, w(S)=v(S)$, and $w$ satisfies the following:

\begin{tabular}{ll}
\hline$S$ & $w(S)$ \\
\hline$\{i, n+1\}$ for $i \in N \backslash\{1\}$ & $\alpha w(\{i\})+\alpha w(\{n+1\})+2(x(N, v)+\delta)$ \\
$\{i, n+2\}$ for $i \in N \backslash\{1\}$ & $\alpha w(\{i\})+\alpha w(\{n+2\})+2(x(N, v)+2 \delta)$ \\
$\{1, n+1\}$ & $\alpha w(\{1\})+\alpha w(\{n+1\})+2\left(x(N, v)+2(n+1)^{2} \delta\right)$ \\
$\{1, n+2\}$ & $\alpha w(\{1\})+\alpha w(\{n+2\})+2(x(N, v)+(2 n+3) \delta)$ \\
$\{n+1, n+2\}$ & $\alpha w(\{n+1\})+\alpha w(\{n+2\})+2\left(x(N, v)+\frac{3}{2} \delta\right)$ \\
$N^{\prime}$ & $\alpha \sum_{i=1}^{n+1} w(\{i\})+(n+1)(x(N, v)+(2 n+2) \delta)$ \\
$N^{\prime \prime}$ & $\alpha \sum_{i=1}^{n+2} w(\{i\})+(n+2)(x(N, v)+(2 n+2) \delta)$ \\
\hline
\end{tabular}


For simplicity, for any $S \subseteq N^{\prime \prime}$, the subgame of $\left(N^{\prime \prime}, w\right)$ induced by $S$ is denoted by $(S, w)$.

From the previous table and from the fact that $\psi$ is equal to $\varphi^{\alpha}$ for all two-player games, we have the following: for $i \in N \backslash\{1\}$,

$$
\begin{aligned}
& \theta_{i}(\{i, n+1\}, w)=\theta_{n+1}(\{i, n+1\}, w)=x(\{i, n+1\}, w)=x(N, v)+\delta \\
& \theta_{i}(\{i, n+2\}, w)=\theta_{n+2}(\{i, n+2\}, w)=x(\{i, n+2\}, w)=x(N, v)+2 \delta
\end{aligned}
$$

and for $i \in\{n+1, n+2\}$,

$$
\theta_{i}(\{n+1, n+2\}, w)=x(\{n+1, n+2\}, w)=x(N, v)+\frac{3 \delta}{2} .
$$

In addition, it holds that

$$
\begin{aligned}
\theta_{1}(\{1, n+1\}, w) & =\theta_{n+1}(\{1, n+1\}, w)=x(\{1, n+1\}, w) \\
& =x(N, v)+2(n+1)^{2} \delta \\
\theta_{1}(\{1, n+2\}, w) & =\theta_{n+2}(\{1, n+2\}, w)=x(\{1, n+2\}, w) \\
& =x(N, v)+(2 n+3) \delta \\
x\left(N^{\prime}, w\right) & =x\left(N^{\prime \prime}, w\right)=x(N, v)+2(n+1) \delta .
\end{aligned}
$$

Together with (7.1) and (7.7),

$$
\begin{aligned}
& \sum_{i=1}^{n+1} \theta_{i}\left(N^{\prime}, w\right)=(n+1) x\left(N^{\prime}, w\right)=(n+1) x(N, v)+2(n+1)^{2} \delta \\
& \sum_{i=1}^{n+2} \theta_{i}\left(N^{\prime \prime}, w\right)=(n+2) x\left(N^{\prime \prime}, w\right)=(n+2) x(N, v)+2(n+1)(n+2) \delta
\end{aligned}
$$

Next, we prove several claims.

Claim $1 \theta_{1}\left(N^{\prime}, w\right)=x(N, v)+2(n+1)^{2} \delta$.

Proof of Claim 1 Suppose that $\theta_{1}\left(N^{\prime}, w\right)>x(N, v)+2(n+1)^{2} \delta$. Since $x(N, v)+$ $2(n+1)^{2} \delta=\theta_{1}(N, v)$ by the definition of $\delta, \theta_{1}\left(N^{\prime}, w\right)>\theta_{1}(N, v)$. Applying population solidarity to $(N, v)$ and $\left(N^{\prime}, w\right)$, it holds that $\theta_{i}\left(N^{\prime}, w\right) \geq \theta_{i}(N, v)$ for all $i \in N$. Then

$$
\sum_{i=1}^{n} \theta_{i}\left(N^{\prime}, w\right)>\sum_{i=1}^{n} \theta_{i}(N, v) .
$$


By (7.8) and (7.1),

$$
\sum_{i=1}^{n} \theta_{i}\left(N^{\prime}, w\right)=(n+1) x(N, v)+2(n+1)^{2} \delta-\theta_{n+1}\left(N^{\prime}, w\right)
$$

and $\sum_{i=1}^{n} \theta_{i}(N, v)=n x(N, v)$. It follows by (7.10) that $(n+1) x(N, v)+2(n+1)^{2} \delta-$ $\theta_{n+1}\left(N^{\prime}, w\right)>n x(N, v)$ or $\theta_{n+1}\left(N^{\prime}, w\right)<x(N, v)+2(n+1)^{2} \delta$. By (7.5), this is equivalent to $\theta_{n+1}\left(N^{\prime}, w\right)<\theta_{n+1}(\{1, n+1\}, w)$. Applying population solidarity to $(\{1, n+1\}, w)$ and $\left(N^{\prime}, w\right)$, it holds that with (7.5) that

$$
\theta_{1}\left(N^{\prime}, w\right) \leq \theta_{1}(\{1, n+1\}, w)=x(N, v)+2(n+1)^{2} \delta
$$

which is a contradiction to the assumption that $\theta_{1}\left(N^{\prime}, w\right)>x(N, v)+2(n+1)^{2} \delta$. If $\theta_{1}\left(N^{\prime}, w\right)<x(N, v)+2(n+1)^{2} \delta$, we can reach a contradiction similarly. Therefore, Claim 1 holds.

Claim $2 \theta_{n+1}\left(N^{\prime}, w\right) \leq x(N, v)+\delta$.

Proof of Claim 2 Suppose that $\theta_{n+1}\left(N^{\prime}, w\right)>x(N, v)+\delta$. By (7.2), we have $\theta_{n+1}\left(N^{\prime}, w\right)>\theta_{n+1}(\{i, n+1\}, w)$ for all $i \in N \backslash\{1\}$. Applying population solidarity to $(\{i, n+1\}, w)$ and $\left(N^{\prime}, w\right)$ for each $i \in N \backslash\{1\}$, we obtain $\theta_{i}\left(N^{\prime}, w\right) \geq$ $\theta_{i}(\{i, n+1\}, w)$ for each $i \in N \backslash\{1\}$. By (7.2), $\theta_{i}\left(N^{\prime}, w\right) \geq x(N, v)+\delta$ for all $i \in N \backslash\{1\}$. All together with Claim 1, we have

$$
\begin{aligned}
\theta_{1}\left(N^{\prime}, w\right) & =x(N, v)+2(n+1)^{2} \delta \\
\theta_{i}\left(N^{\prime}, w\right) & \geq x(N, v)+\delta \quad \text { for all } i \in N \backslash\{1\} \\
\theta_{n+1}\left(N^{\prime}, w\right) & >x(N, v)+\delta,
\end{aligned}
$$

and so $\sum_{i=1}^{n+1} \theta_{i}\left(N^{\prime}, w\right)>(n+1) x(N, v)+2(n+1)^{2} \delta+n \delta$, which contradicts (7.8). Therefore, Claim 2 holds.

Claim $3 \theta_{i}\left(N^{\prime \prime}, w\right)>\theta_{i}\left(N^{\prime}, w\right)$ for some $i \in N^{\prime}$.

Proof of Claim 3 Suppose that $\theta_{i}\left(N^{\prime \prime}, w\right) \leq \theta_{i}\left(N^{\prime}, w\right)$ for all $i \in N^{\prime}$. Then

$$
\begin{aligned}
\theta_{n+1}\left(N^{\prime \prime}, w\right) & \leq \theta_{n+1}\left(N^{\prime}, w\right) \leq x(N, v)+\delta<x(N, v)+\frac{3}{2} \delta \\
& =\theta_{n+1}(\{n+1, n+2\}, w),
\end{aligned}
$$

where the second inequality follows from Claim 2, and the last equality follows from (7.4). Applying population solidarity to $(\{n+1, n+2\}, w)$ and $\left(N^{\prime \prime}, w\right)$, we have $\theta_{n+2}\left(N^{\prime \prime}, w\right) \leq \theta_{n+2}(\{n+1, n+2\}, w)$. Since, from (7.3) and (7.4), for all $i \in$ $N \backslash\{1\}$,

$$
\begin{aligned}
\theta_{n+2}\left(N^{\prime \prime}, w\right) & \leq \theta_{n+2}(\{n+1, n+2\}, w)=x(N, v)+\frac{3}{2} \delta<x(N, v)+2 \delta \\
& =\theta_{n+2}(\{i, n+2\}, w),
\end{aligned}
$$


it follows that for all $i \in N \backslash\{1\}, \theta_{n+2}\left(N^{\prime \prime}, w\right)<\theta_{n+2}(\{i, n+2\}, w)$. Applying population solidarity to $(\{i, n+2\}, w)$ and $\left(N^{\prime \prime}, w\right)$, it holds that $\theta_{i}\left(N^{\prime \prime}, w\right) \leq$ $\theta_{i}(\{i, n+2\}, w)$ for all $i \in N \backslash\{1\}$. By (7.3), $\theta_{i}\left(N^{\prime \prime}, w\right) \leq x(N, v)+2 \delta$ for all $i \in N \backslash\{1\}$. All together with Claim 1, we have

$$
\begin{aligned}
\theta_{1}\left(N^{\prime \prime}, w\right) & \leq \theta_{1}\left(N^{\prime}, w\right)=x(N, v)+2(n+1)^{2} \delta \\
\theta_{i}\left(N^{\prime \prime}, w\right) & \leq x(N, v)+2 \delta \text { for all } i \in N \backslash\{1\} \\
\theta_{n+1}\left(N^{\prime \prime}, w\right) & \leq x(N, v)+\delta \\
\theta_{n+2}\left(N^{\prime \prime}, w\right) & \leq x(N, v)+\frac{3}{2} \delta .
\end{aligned}
$$

Then $\sum_{i=1}^{n+2} \theta_{i}\left(N^{\prime \prime}, w\right) \leq\left(x(N, v)+2(n+1)^{2} \delta\right)+(n-1)(x(N, v)+2 \delta)+(x(N, v)+$ $\delta)+\left(x(N, v)+\frac{3}{2} \delta\right)$, and so

$$
\begin{aligned}
\sum_{i=1}^{n+2} \theta_{i}\left(N^{\prime \prime}, w\right) & \leq(n+2) x(N, v)+\left(2 n^{2}+6 n+\frac{5}{2}\right) \delta \\
& <(n+2) x(N, v)+2(n+1)(n+2) \delta
\end{aligned}
$$

which yields a contradiction to (7.9). Therefore, Claim 3 holds.

Claim $4 \theta_{i}\left(N^{\prime \prime}, w\right)<\theta_{i}\left(N^{\prime}, w\right)$ for some $i \in N^{\prime}$.

Proof of Claim 4 Suppose that $\theta_{i}\left(N^{\prime \prime}, w\right) \geq \theta_{i}\left(N^{\prime}, w\right)$ for all $i \in N^{\prime}$. Then

$$
\begin{aligned}
\theta_{1}\left(N^{\prime \prime}, w\right) & \geq \theta_{1}\left(N^{\prime}, w\right)=x(N, v)+2(n+1)^{2} \delta>x(N, v)+(2 n+3) \delta \\
& =\theta_{1}(\{1, n+2\}, w),
\end{aligned}
$$

where the second equality follows from Claim 1, and the last equality follows from (7.6). Applying population solidarity to $(\{1, n+2\}, w)$ and $\left(N^{\prime \prime}, w\right)$, we have $\theta_{n+2}\left(N^{\prime \prime}, w\right) \geq \theta_{n+2}(\{1, n+2\}, w)$. Then

$\theta_{n+2}\left(N^{\prime \prime}, w\right) \geq \theta_{n+2}(\{1, n+2\}, w)=x(N, v)+(2 n+3) \delta>x(N, v)+2(n+1) \delta$,

where the second equality follows from (7.6). Since $\theta_{i}\left(N^{\prime \prime}, w\right) \geq \theta_{i}\left(N^{\prime}, w\right)$ for all $i \in N^{\prime}$,

$$
\sum_{i=1}^{n+1} \theta_{i}\left(N^{\prime \prime}, w\right)+\theta_{n+2}\left(N^{\prime \prime}, w\right)>\sum_{i=1}^{n+1} \theta_{i}\left(N^{\prime}, w\right)+(x(N, v)+2(n+1) \delta) .
$$

By (7.8) and (7.9),

$$
\begin{aligned}
(n+2) x(N, v)+2(n+1)(n+2) \delta> & (n+1) x(N, v)+2(n+1)^{2} \delta \\
& +(x(N, v)+2(n+1) \delta),
\end{aligned}
$$


where the right side is $(n+2) x(N, v)+2(n+1)(n+2) \delta$, which is a contradiction. Therefore, Claim 4 holds.

From Claims 3 and 4 , we reach a contradiction to population solidarity applied to $\left(N^{\prime}, w\right)$ and $\left(N^{\prime \prime}, w\right)$. It completes the proof of Theorem 5.2.

\section{References}

Chun, Y. (1986). The solidarity axiom for quasi-linear social choice problems. Social Choice and Welfare, 3, 297-310.

Chun, Y., \& Park, B. (2012). Population solidarity, population fair-ranking, and the egalitarian value. International Journal of Game Theory, 41, 255-270.

Driessen, T. S. H., \& Funaki, Y. (1991). Coincidence of and collinearity between game theoretic solutions. OR Spektrum, 13, 15-30.

Funaki, Y. (1998). Dual axiomatizations of solutions of cooperative games, mimeo.

Funaki, Y., \& Yamato, T. (2001). The core and consistency properties: a general characterisation. International Game Theory Review, 3, 175-187.

Hart, S., \& Mas-Colell, A. (1988). The potential of the Shapley value. In A. E. Roth (Ed.), The Shapley value. Essays in honor of L.S. Shapley (pp. 127-137). Cambridge: Cambridge University Press.

Hart, S., \& Mas-Colell, A. (1989). Potential, value and consistency. Econometrica, 57, 589-614.

Joosten, R. (1996). Dynamics, equilibria and values. Dissertation, Maastricht University.

Ju, Y., Borm, P. E. M., \& Ruys, P. H. M. (2007). The consensus value: a new solution concept for cooperative games. Social Choice and Welfare, 28, 685-703.

Levinský, R., \& Silársky, P. (2004). Global monotonicity of values of cooperative games: an argument supporting the explanatory power of Shapley's approach. Homo Oeconomicus, 20, 473-492.

Oishi,T., Nakayama, M., Hokari, T., \& Funaki, Y. (2012). Anti-duality in TU-games applied to solutions, axioms, and axiomatizations, mimeo.

Oishi, T., Nakayama, M., Hokari, T., \& Funaki, Y. (2016). Duality and anti-duality in TU games applied to solutions, axioms, and axiomatizations, Journal of Mathematical Economics 63, 43-53.

Peleg, B., \& Sudhölter, P. (2003). Introduction to the theory of cooperative games. Boston: Kluwer Academic.

Shapley, L. S. (1953). A value for $n$-person games. In H. W. Kuhn \& A. W. Tucker (Eds.), Annals of mathematics studies 28 (contributions to the theory of games vol. 2) (pp. 307-317). Princeton: Princeton University Press.

Sobolev, A.I. (1973). The functional equations that give the payoffs of the players in an $n$-person game. In: E. Vilkas (Ed.), Advaces in game theory, Izdat. "Mintis", Vilnius (pp. 151-153) (in Russion).

Thomson, W. (1983). Problems of fair division and the egalitarian solution. Journal of Economic Theory, 31, 211-226.

van den Brink, R. (2001). An axiomatization of the Shapley value using a fairness property. International Journal of Game Theory, 30, 309-319.

van den Brink, R. (2007). Null players or nullifying players: the difference between the Shapley value and equal division solutions. Journal of Economic Theory, 136, 767-775.

van den Brink, R., \& Funaki, Y. (2009). Axiomatizations of a class of equal surplus sharing solutions for TU-games. Theory and Decision, 67, 303-340.

van den Brink, R., Funaki, Y., \& Ju, Y. (2013). Reconciling marginalism with egalitarianism: consistency, monotonicity, and implementation of egalitarian Shapley values. Social Choice and Welfare, 40, 693714. 